\title{
Anatomical Variations of the Saphenous Nerve in the Adductor Canal
}

Rutvik Vanamala ${ }^{1}$, Niels Hammer ${ }^{2}$, Scheherezade Soltani ${ }^{3}$, Khoon Lim ${ }^{4}$, Michael Charles Wyatt ${ }^{4}$ and David Christopher Kieser ${ }^{4 *}$

*Correspondence: kieserdavid@gmail.com

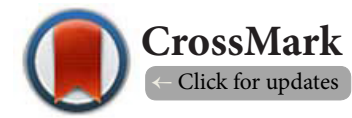

'Department of Orthopaedics, MidCentral District Health Board, New Zealand.
2Department of Anatomy, University of Otago, Dunedin, New Zealand.
${ }^{3}$ Oxford University NHS Foundation Trust, Division of Spinal Surgery, Oxford, England.
${ }^{4}$ Department of Orthopaedic Surgery and Musculoskeletal Medicine, University of Otago, Christchurch, New Zealand.

\begin{abstract}
Local anaesthetic blockage of the saphenous nervewithin the adductor canal block hasevolved asa popular analgesic technique for operations around the knee. However, not all patients benefit equally, despite accurate placement of local anaesthetic into the adductor canal under ultrasound guidance. It has been proposedthat anatomical variations of the saphenous nerve within the canal may account for the variable clinical efficacy of such blocks. Thus, we performed a dissection study of 22 cadaveric knees to determine the variance of the saphenous nerve. This study revealed multiple variations of the saphenous nerve within the adductor canal. However, despite these anatomical variations, an accurately placed adductor canal block should provide adequate anaesthesia of the saphenous nerve and its branches.
\end{abstract}

Keywords: Nerve, knee, saphenous, surgery

\section{Introduction}

Postoperative pain following operations around the knee, particularly total knee joint replacement, has been a major challenge [1]. This pain affectsearly mobility, patient participation in rehabilitation and overall patient experience [1]. Thus, optimal analgesia is essential to improve early function and satisfaction.

A variety of analgesic modalities are available, including systemic analgesia and local topical therapies, such as icing, compression and bracing. However, for enhanced recovery after surgery, avoidance of systemic analgesics, particularly opiates, is encouraged [2]. Furthermore, a full range of motion without the mechanical limitations of bracing is necessary.

Peripheral nerve blocks have therefore been advocated to limit systemic analgesia and preserve range of motion [2]. An adductor canal block, which involves a local anaesthetic injection or infusion into the adductor canal is commonly performed [2]. This is typically performed under ultrasound guidance and has been shown to enhance early ambulation and improve early postoperative outcomes [2]. The adductor canal, also known as the subsartorial or 'Hunter's' canal, is an aponeurotic intermuscular tunnel in the thigh that extends from the apex of the femoral tunnel until the adductor hiatus and contains the femoral artery, femoral vein, posterior branch of the obturator nerve and branches of the femoral nerve, namely the saphenous nerve and nerve to vastus medialis (NVM) [3].

The saphenous nerve is thought to contribute significantly to nociception of the knee. Itenters the adductor canal immediately lateral to the femoral artery at the apex of femoral triangle [4]. During its course in the adductor canal it passes anterior to the femoral artery to reach its medial side [4]. It then bifurcates into the infrapatellar branch and the main sartorial branch. The infrapatellar branchthen pierces the sartorius and fascia lata to be distributed to the skin of the anterior knee. In contrast, the sartorial branchleaves the canal, along with the descending genicular artery, by exiting beneath the posterior border of the sartorius where it pierces fascia lata to reach the subcutaneous tissue and accompany the saphenous vein as it descends the leg.

An adductor canal blockis technically easy to perform and believed to provide a blockade of the two main branches of the saphenous nerve [5]. However, despite radiological or ultrasound guidance ensuring accurate placement of the local anaesthetic into the adductor canal, the effectiveness of patients' postoperative pain relief varies. It has been proposed therefore that anatomical variationsof the saphenous nerve account for such variancesin clinical efficacy.

The aim of this study is therefore to assess the saphenous 
nerve variation within the adductor canal and determine if anatomical variations are likely to be the cause of the variance in analgesia experienced by patients.

\section{Methods}

The Human Ethics Committee of the University of Otago approvedthis study (H17/006). Twenty-twoethanol-phenoxyethanolembalmed cadaveric lower limbs were procured for this study. Cases with prior trauma or surgical intervention to the knee were excluded. The skin and subcutaneous tissue of the anterior and medial thigh, knee and leg were dissected under loupe magnification to identify the infrapatellar and sartorial branch of the saphenous nerve, as well as the sartorius and vastus medialis muscle. A plane was then developed between these two muscles to reveal the neurovascular structures of the adductor canal. The adductor canal was divided into its proximal, middle and distal thirds. The course and distribution of the saphenous nerve proper and its branches was then assessed according to its location within the canal.

\section{Results}

All 22samples were utilised. These included 13 female ( 3 bilateral) and 5 male ( 1 bilateral) cadavers. Fourteen were left-sided and eightwere right-sided. The average age of the cadavers was 83 years (range 62 to 98 years). All cadavers were of New Zealand European ancestry.

In all samples the saphenous nerve was identified within the adductor canal. In 17 samples, the bifurcation of the saphenous nerve occurred in the distal third of the adductor canal, whereas in four cadavers the bifurcation occurred in the middle third of the adductor canal. None bifurcated in the proximal third (Figure 1).

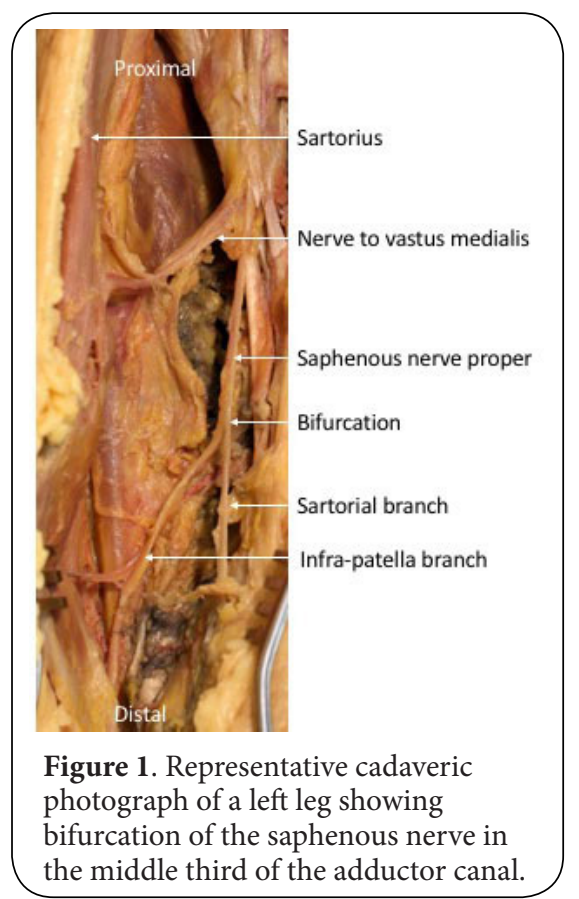

One sample had a common trunk with the NVM. This common trunk divided into the saphenous nerve and NVM in the proximal third of the adductor canal. The saphenous nerve then failed to bifurcate and continued directly as the sartorial branch without giving off an infrapatellar branch (Figure 2).

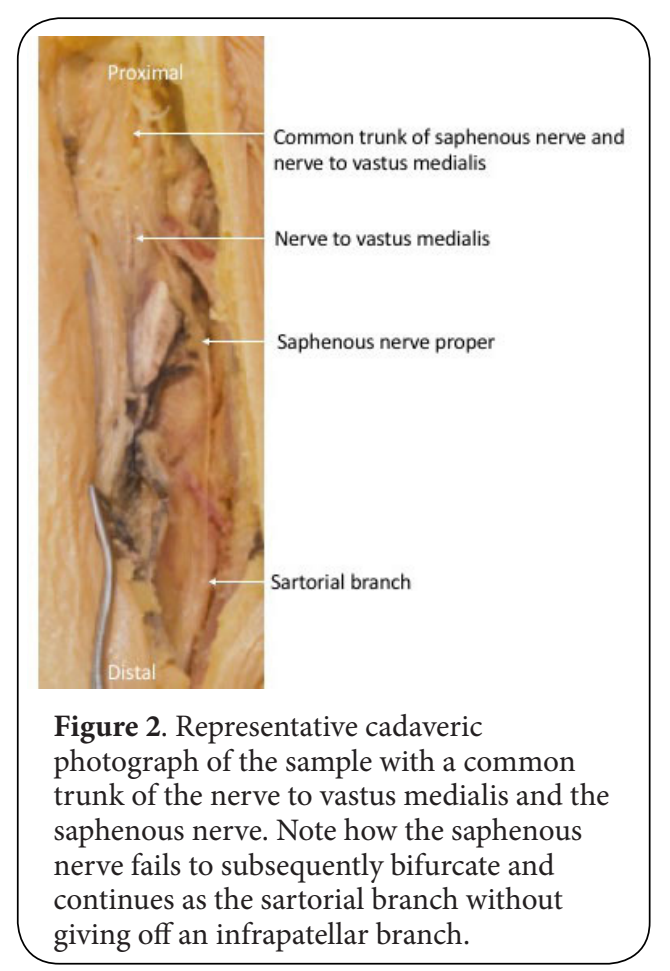

The sartorial branch of the saphenous nerve was identified in the distal third of the canal in all samples. Similarly, the infrapatellar branch of the saphenous nerve was identified within the distal third of the canal in all samples except the one sample with a common trunk of the NVM and saphenous nerve, which did not have an infrapatellar branch.

\section{Discussion}

To our knowledge, this is the first study to analyse the variations of the saphenous nerve within the adductor canal. We identified significant variations of the saphenous nerve, but found that all samples had a saphenous nerve within the adductor canal. Furthermore, all samples bifurcated into the infrapatellar and sartorial branches in the distal two thirds of the canal, except one sample that did not bifurcate.

Typically adductor canal blocks are performed in the distal third of the adductor canal, where the vascular structures dive posteriorly away from the saphenous nerve $[6,7]$. This results in a lower risk of vascular injury. In our study allsaphenous nerves that bifurcateddid so in the middle or distal thirds of the canal and all branches of the saphenous nerve were identified in the distal third of the canal. Furthermore, it is well recognised that local anaesthetic spreads proximally 
in the adductor canal $[6,7]$. Thus, our results would suggest that successful anaesthesia of the saphenous nerve and its branches is likely with an adductor canal block in the distal third of the adductor canal (Figure 3).

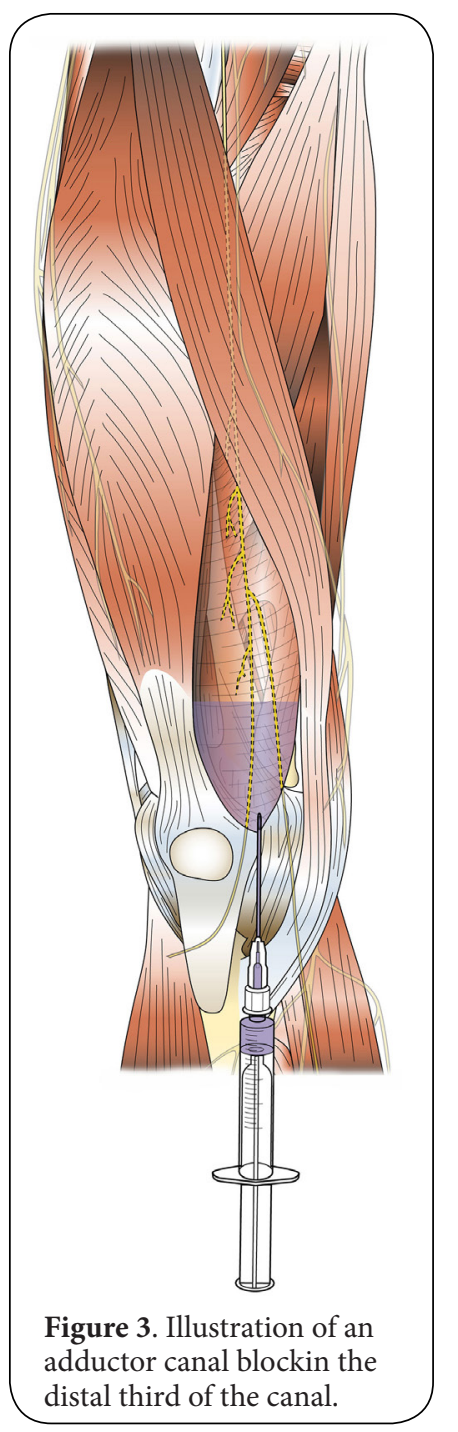

These findings therefore suggest that, despite anatomical variation of the saphenous nerve occurring within the adductor canal, an accurately placed adductor canal block should provide reliable blockade of the saphenous nerve and its branches. In only one case could a reduced anaesthetic effect be attributed to anatomical variation and this was a case of a combined trunk of the nerve to vastus medialis and the saphenous nerve, that subsequently failed to give off an infrapatellar branch. Thus, consideration of the contribution of the posterior tibial, femoral and peroneal nerves to the knee joint as well as the innervation of the surrounding soft tissues should be given when understanding the variance in patient analgesia with adductor canal blocks.

This study has a number of limitations. Firstly, its low numbers may result in rare anatomical variations not being identified. Secondly,the cadaveric dissection approach utilised does not analyse the efficacy of an adductor canal block to bathe the nerves in local anaesthetic, but rather analyses the gross course and distribution of the nerve and its branches. Further analysis of the ease at which local anaesthetic disperses within the canal is necessary. Lastly, inference of analgesia is based on the anatomical reliability of the saphenous nerve, but the degree of its articular and periarticular innervation is not provided.

In conclusion, there are multiple variations of saphenous nerve within the adductor canal. However, despite these anatomical variations, an accurately placed adductor canal block should provide adequate anaesthesia of the saphenous nerve and its branches.

\section{Competing interests}

The authors declare that they have no competing interests.

Authors' contributions

\begin{tabular}{|l|c|c|c|c|c|c|}
\hline Authors' contributions & RV & NH & SS & KL & MCW & DCK \\
\hline Research concept and design & -- & $\checkmark$ & -- & -- & -- & $\checkmark$ \\
\hline Collection and/or assembly of data & $\checkmark$ & $\checkmark$ & -- & -- & -- & $\checkmark$ \\
\hline Data analysis and interpretation & $\checkmark$ & $\checkmark$ & $\checkmark$ & $\checkmark$ & $\checkmark$ & $\checkmark$ \\
\hline Writing the article & $\checkmark$ & $\checkmark$ & -- & -- & -- & $\checkmark$ \\
\hline Critical revision of the article & -- & $\checkmark$ & $\checkmark$ & $\checkmark$ & $\checkmark$ & $\checkmark$ \\
\hline Final approval of article & $\checkmark$ & $\checkmark$ & $\checkmark$ & $\checkmark$ & $\checkmark$ & $\checkmark$ \\
\hline Statistical analysis & -- & $\checkmark$ & -- & -- & -- & $\checkmark$ \\
\hline
\end{tabular}

Acknowledgment

Glynny Kieser for her editorial input. Christine Hammer for her photographic input.

Publication history

EIC: D. John Doyle, Case Western Reserve University, USA.

Received: 09-Aug-2019 Final Revised:06-Sept-2019

Accepted: 09-Sept-2019 Published: 25-Sep-2019

\section{References}

1. Vendittoli PA, Makinen P, Drolet P, Lavigne $M$, Fallaha $M$, Guertin $M C$ and Varin F. A multimodal analgesia protocol for total knee arthroplasty. A randomized, controlled study. J Bone Joint Surg Am. 2006; 88:282-9. I Article | PubMed

2. Hanson NA, Allen CJ, Hostetter LS, Nagy R, Derby RE, Slee AE, Arslan A and Auyong DB. Continuous ultrasound-guided adductor canal block for total knee arthroplasty: a randomized, double-blind trial. Anesth Analg. 2014; 118:1370-7. | Article | PubMed

3. Sauerland EK, Tank PW. Grant's dissector. Lippincott Williams \& Wilkins. 2005; 128.

4. Horn JL, Pitsch T, Salinas F and Benninger B. Anatomic basis to the ultrasound-guided approach for saphenous nerve blockade. Reg Anesth Pain Med. 2009; 34:486-9. | Article | PubMed

5. Manickam B, Perlas A, Duggan E, Brull R, Chan VW and Ramlogan R. Feasibility and efficacy of ultrasound-guided block of the saphenous nerve in the adductor canal. Reg Anesth Pain Med. 2009; 34:578-80. I PubMed

6. Jenstrup MT, Jaeger P, Lund J, Fomsgaard JS, Bache S, Mathiesen O, Larsen TK and Dahl JB. Effects of adductor-canal-blockade on pain and ambulation after total knee arthroplasty: a randomized study. Acta 
Vanamala et al. Journal of Anesthesiology \& Clinical Science 2019,

http://www.hoajonline.com/journals/pdf/2049-9752-8-2.pdf

Anaesthesiol Scand. 2012; 56:357-64. | Article | PubMed

7. Kwofie MK, Shastri UD, Gadsden JC, Sinha SK, Abrams JH, Xu D and Salviz EA. The effects of ultrasound-guided adductor canal block versus femoral nerve block on quadriceps strength and fall risk: a blinded, randomized trial of volunteers. Reg Anesth Pain Med. 2013; 38:321-5. I Article | PubMed

\section{Citation:}

Vanamala R, Hammer N, Soltani S, Lim K, Wyatt MC and Kieser DC. Anatomical Variations of the Saphenous

Nerve in the Adductor Canal. J Anesthesiol Clin Sci. 2019; 8:2. http://dx.doi.org/10.7243/2049-9752-8-2 